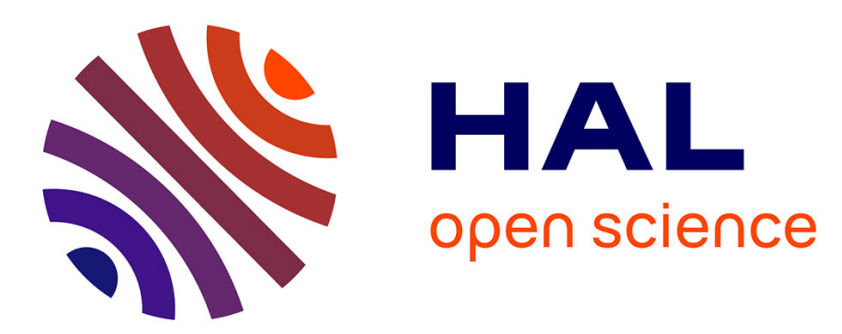

\title{
Analyse confirmatoire de la version courte de la Center for Epidemiological Studies of Depression Scale (CES-D10) chez les adolescents
}

\author{
Nathalie Cartierre, Nathalie . Coulon, René Demerval
}

\section{- To cite this version:}

Nathalie Cartierre, Nathalie . Coulon, René Demerval. Analyse confirmatoire de la version courte de la Center for Epidemiological Studies of Depression Scale (CES-D10) chez les adolescents. L'Encéphale, 2011, 37, pp.273-277. 10.1016/j.encep.2011.01.011 . hal-01353836

\section{HAL Id: hal-01353836 \\ https://u-bourgogne.hal.science/hal-01353836}

Submitted on 2 Nov 2016

HAL is a multi-disciplinary open access archive for the deposit and dissemination of scientific research documents, whether they are published or not. The documents may come from teaching and research institutions in France or abroad, or from public or private research centers.
L'archive ouverte pluridisciplinaire HAL, est destinée au dépôt et à la diffusion de documents scientifiques de niveau recherche, publiés ou non, émanant des établissements d'enseignement et de recherche français ou étrangers, des laboratoires publics ou privés. 
Titre : Analyse confirmatoire de la version courte de la Center for Epidemiological Studies of Depression Scale (CES-D10) chez les adolescents

Auteurs : Nathalie Cartierre, Nathalie Coulon, René Demerval

UPRES URECA EA 1059, Equipe « Famille, Santé \& Emotion », Université de Lille 3

\section{Citation :}

Cartierre, N., Coulon, N., \& Demerval, R. (2011). Analyse confirmatoire de la version courte de la Center for Epidemiological Studies of Depression Scale (CES-D10) chez les adolescents. Encéphale, 37, 273-277.

Auteur correspondant : Nathalie Cartierre, Université de Bourgogne nathalie.cartierre@u-bourgogne.fr 
Résumé: La CES-D (Center for Epidemiological Studies-Depression Scale) est un outil d'évaluation de la symptomatologie dépressive. Différentes études ont été réalisées sur la version courte en 10 items, y compris chez les adolescents. Elles ont mis en évidence une structure en deux facteurs Affect négatif (huit items) et Affect positif (deux items). L'objet de la présente étude est de valider la version française de la CES-D10 sur un échantillon de 269 adolescents et de tester une structure uni-factorielle en tenant compte d'un artefact méthodologique supposé sur le facteur Affect positif. Deux analyses ont été réalisées : une analyse factorielle confirmatoire afin de choisir le modèle présentant le meilleur ajustement suivie d'une analyse confirmatoire multi-groupes sur la structure retenue permettant ainsi de tester son invariance selon le genre. Les résultats montrent que la CES-D10 présente une consistance interne acceptable. Le modèle le mieux ajusté aux données est celui à un seul facteur avec corrélation des erreurs pour les deux items associés au facteur Affect positif dans de précédentes recherches. L'invariance structurelle selon le genre est confortée ce qui permet d'éviter un biais d'interprétation des différences constatées entre les filles et les garçons.

Mots-clés : Adolescents ; Échelle ; Dépression ; CES-D10 ; Analyse factorielle confirmatoire 
Confirmatory factor analysis of the short French version of the Center for Epidemiological Studies of Depression Scale (CES-D10) in adolescents

Keywords: Adolescents; Scale; Depression; CES-D10; Confirmatory factor analysis

Background. - Screening depressivity among adolescents is a key public health priority. In order to measure the severity of depressive symptomatology, a four-dimensional 20 items scale called "Center for Epidemiological Studies-Depression Scale" (CES-D) was developed. A shorter 10-item version was developed and validated (Andresen et al. [Am J Prev Med 10 (1994) 77-84]). For this brief version, several authors supported a two factor structure Negative and Positive affect - but the relationship between the two reversed-worded items of the Positive affect factor could be better accounted for by correlated errors.

Objectives. - The aim of this study is triple: firstly to test a French version of the CES-D10 among adolescents; secondly to test the relevance of a one-dimensional structure by considering error correlation for Positive affect items; finally to examine the extent to which this structural model is invariant across gender.

Method. - The sample was composed of 269 French middle school adolescents (139 girls and 130 boys, mean age: $13.8, \mathrm{SD}=0.65)$. Confirmatory Factorial Analyses $(\mathrm{CFA})$ using the LISREL 8.52 were conducted in order to assess the adjustment to the data of three factor models: a one-factor model, a two-factor model (Positive and Negative affect) and a onefactor model with specification of correlated errors between the two reverse-worded items. Then, multi-group analysis was conducted to test the scale invariance for girls and boys.

Results. - Internal consistency of the CES-D10 was satisfying for the adolescent sample $(\alpha=$ .75). The best fitting model is the one-factor model with correlated errors between the two items of the previous Positive affect factor $\left(\chi^{2} / \mathrm{dl}=2.50\right.$; GFI $=0.939 ; \mathrm{CFI}=0.894$; RMSEA $=.076)$. This model presented a better statistical fit to the data than the one-factor model without error correlation: $\chi^{2}$ diff $(1)=22.14, p<.001$. Then, the one factor model with correlated errors was analyzed across separate samples of girls and boys. The model explains the data somewhat better for boys than for girls. The model's overall $\chi^{2}(68)$ without equality constraints from the multi-group analysis was 107.98 . The $\chi^{2}(89)$ statistic for the model with equality-constrained factor loadings was 121.31 . The change in the overall chi-square is not statistically significant. This result implies that the model is therefore invariant across gender. The mean scores were higher for girls than boys: 9.69 vs $7.19 ; \mathrm{t}(267)=4.13, p<.001$.

Conclusions. - To conclude, and waiting for other researches using the French version of CES-D10 for adolescents, it appears that this short scale is generally acceptable and can be a useful tool for both research and practice. The scale invariance across gender has been demonstrated but the invariance across age must be tested too. 
Introduction

A l'adolescence, les troubles dépressifs augmentent de façon remarquable et sont les troubles les plus présents. Ils sont associés au comportement suicidaire [29] et présentent un risque de persistance à l'âge adulte et de chronicisation [20]. Le dépistage de la symptomatologie dépressive chez les adolescents est donc un enjeu important de santé publique. Différents outils sont disponibles parmi lesquels la Center for Epidemiological Studies-Depression Scale [CES-D ; 21] qui mesure la sévérité de la dépressivité sur un continuum allant du bien-être psychologique total à l'état dépressif grave. La CES-D est également utilisée pour estimer la prévalence des troubles dépressifs dans la population générale. Cette échelle en 20 items a été largement diffusée et traduite en plusieurs langues, dont le français [11]. La CES-D a fait l'objet de validations chez les adultes et les personnes âgées, mais également chez les adolescents [7, 12, 22]. Différentes versions courtes ont été développées dont notamment la CES-D10, version en 10 items proposée par Andresen et al. [1].

Les qualités psychométriques la CES-D en 20 items sont bonnes avec une structure factorielle en quatre dimensions - Affect dépressif, Affect positif, Troubles somatiques, Problèmes interpersonnels - confirmée pour différentes populations [7, 15, 21]. La CES-D10 présente une bonne cohérence interne, comparable à celle de l'échelle originale. Par contre, la composante Problèmes interpersonnels disparaît de la structure factorielle. Certains auteurs ont ainsi montré des résultats en faveur d'un modèle à trois facteurs, et d'autres défendent une structure à deux facteurs, chez des personnes âgées [8, 17]. La solution à deux facteurs Affect négatif et Affect positif - correspond à un regroupement des items attribués à Affect dépressif et Troubles somatiques. Ce modèle bi-dimensionnel est aussi retrouvé pour une population adolescente [4]. Cependant, cette structure à deux facteurs est elle-même discutée.

Les critiques formulées se situent sur trois plans. Au niveau statistique, le facteur Affect positif est composé de seulement deux items, ce qui met en doute sa fiabilité. Sur le plan du contenu, ces deux items sont formulés avec une orientation positive ( "j’ai été heureux(euse) » et «j'ai été confiant en l'avenir ») alors que les huit autres items le sont avec une orientation négative. L'émergence d'un facteur spécifique peut être tout simplement liée à un artefact [15]. Enfin, au niveau théorique, le fondement même d'une distinction entre deux formes différentes d'affect - positive et négative - est l'objet de controverses [3, 10]. Par ailleurs, comme l'indiquait Radloff pour la CES-D originale [21, p. 398], «The high internal consistency of the scale found in all groups argues against undue emphasis on separate factors.». Quoiqu'il 
en soit, il est essentiel de prendre en compte l'usage qui est fait de l'échelle, en 10 ou 20 items, selon qu'il s'agit d'effectuer un diagnostic clinique, d'évaluer l'évolution de la prévalence dans une population ou encore de réaliser la première phase d'un dépistage.

A l'adolescence, les taux de dépression sont déjà clairement plus importants chez les filles que chez les garçons [19]. Cette différenciation liée au genre pose la question de l'invariance structurelle des outils de mesure permettant de légitimer la comparaison de ces deux groupes. Certains auteurs ont noté, dans le cas de la CES-D20, un biais structurel dû à certains items « connotés» $[2,6,26]$, notamment J'ai eu des crises de larmes et J'ai parlé moins que d'habitude. Les items incriminés n'apparaissent d'ailleurs plus dans la quasi totalité des versions courtes. L'invariance structurelle de la CES-D10 selon le genre a été confirmée chez les personnes âgées [17] mais peu de données existent concernant les adolescents.

Trois objectifs sont visés dans la recherche présente. Le premier concerne la validation d'une version courte de la CES-D en langue française pour une population adolescente. Le deuxième objectif concerne la structure factorielle de la CES-D10. Selon nous, la pertinence d'une version courte réside dans son utilisation à des fins épidémiologiques et, dans ce cas, à moins de constater un défaut d'ajustement du modèle unidimensionnel, l'apport d'une structure multifactorielle n'est pas déterminant. Comme d'autres auteurs, nous pensons que les relations entre les deux items à orientation positive peuvent être prises en compte, non pas par un facteur spécifique, mais en autorisant la covariation de leurs erreurs de mesure [28]. Enfin, il est primordial de vérifier, par le biais d'une analyse multi-groupes, que la mesure effectuée chez les filles et les garçons repose sur les mêmes construits psychologiques.

\section{Méthode}

\section{Participants et procédure}

La population est composée de 289 adolescents et adolescentes en classe de quatrième dans quatre collèges du département du Nord de la France. Deux collèges sont en milieu rural et deux en milieu urbain. Les données exploitables statistiquement ont concerné 269 participants, d'âge moyen 13,8 ans (écart-type $=0,65$ ), dont 51,5\% de filles. Parmi les participants, 24,48\% ont redoublé une classe, 67,47\% vivent dans une famille nucléaire et $15,57 \%$ dans une famille monoparentale. 
Outil

La Center for Epidemiological Studies-Depression Scale, version courte CES-D10

Nous avons utilisé la version courte en 10 items de la CES-D (voir annexe), validée chez les personnes âgées par Andresen et al. [1] puis chez les adolescents [4]. La traduction française de l'échelle est celle validée par Fuhrer et Rouillon [11] pour la version originale en 20 items. L'adolescent est interrogé sur la fréquence avec laquelle il a ressenti différents symptômes durant la semaine écoulée. Pour chacun des 10 items, les quatre modalités de réponse étaient ensuite cotées de 0 à 3 (de la perception la plus négative à la plus positive).

Analyse des données

Deux types d'analyses factorielles confirmatoires ont été réalisées successivement à l'aide du logiciel LISREL 8.52 via des estimations par le maximum de vraisemblance. Nous avons d'abord confronté trois structures factorielles sur l'échantillon complet. Le premier modèle teste l'ajustement d'une structure à un seul facteur, le deuxième examine la solution à deux facteurs - Affect négatif et Affect positif - et le dernier reprend la solution à un facteur mais en corrélant les erreurs associées aux deux items 5 et 8 attribués au facteur Affect positif. Ensuite, le modèle le mieux ajusté a été l'objet d'une analyse confirmatoire multi-groupes afin de tester l'invariance de structure entre les filles et les garçons.

Pour ces analyses, plusieurs indices d'ajustement ont été utilisés. Le $\chi^{2}$ étant très sensible à la taille des échantillons, certains auteurs se réfèrent au $\chi^{2} / \mathrm{dl}$ et considèrent qu'une valeur entre 2 et 3 indique un ajustement acceptable [16]. Le Root Mean Squared Error of Approximation (RMSEA) constitue un indice de qualité d'ajustement du modèle : on considère généralement qu'en dessous de 0,05, la qualité est bonne ; entre 0,05 et 0,08, elle est acceptable [5]. Pour les indices de comparaison, Comparative Fit Index (CFI) et Goodness of Fit Index (GFI), il est souhaitable qu'ils soient supérieurs à 0,90 et même 0,95 [14].

Résultats

Analyse structurelle 
La CES-D10 présente une cohérence interne satisfaisante $(\alpha=0,75)$. La cohérence est également satisfaisante $(\alpha=0,76)$ pour le facteur Affect négatif qui regroupe les items 1, 2, 3, 4, 6, 7, 9 et 10. Par contre, le facteur Affect positif, composé des items 5 et 8, présente une cohérence interne très insuffisante $(\alpha=0,38)$.

La première analyse confirmatoire permet de confronter les indices d'ajustement de trois modèles structurels (tableau 1) : le modèle avec une seule variable latente, le modèle avec deux variables latentes et enfin le modèle avec une variable latente et la corrélation des erreurs associées aux items 5 et 8 . Il apparaît clairement que le modèle à deux facteurs est à écarter : les indices d'ajustement s'éloignent nettement des recommandations habituelles. Le meilleur modèle est unidimensionnel avec covariation des erreurs pour les items 5 et 8 dont l'orientation des réponses est positive contrairement aux huit autres items. Le gain dans l'ajustement par rapport au premier modèle, donc sans corrélation, est significatif : $\chi^{2}$ diff $(1)=$ $22,14, p<0,001$. L'ajustement pourrait être encore amélioré en ajoutant un lien de covariation entre les erreurs associées aux items 10 et $4: \chi^{2} / \mathrm{dl}=1,88$, RMSEA $=0,057$, GFI $=0,956$ et $\mathrm{CFI}=0,956$. Cette solution, suggérée par les indices de modification fournis par LISREL, ne sera pas retenue pour la suite des analyses car cette modification technique n'est pas suffisamment étayée au niveau conceptuel.

Tableau 1. Indices d'ajustement pour les trois modèles structuraux $(n=269)$

\begin{tabular}{lcccccc}
\hline Modèles & $\chi^{2}$ & $\mathrm{dl}$ & $\chi^{2 / \mathrm{dl}}$ & RMSEA & GFI & CFI \\
\hline Un facteur & 107,340 & 35 & 3,067 & 0,088 & 0,925 & 0,851 \\
Deux facteurs & 147,226 & 34 & 4,330 & 0,108 & 0,905 & 0,766 \\
Un facteur avec erreurs corrélées (5-8) & 85,196 & 34 & 2,505 & 0,076 & 0,939 & 0,894 \\
\hline
\end{tabular}

Analyse confirmatoire multi-groupes

L'analyse multi-groupes respecte les étapes définies par Kline [16]. Quatre analyses ont été réalisées en reprenant le modèle qui présentait le meilleur ajustement. Les deux premières sont effectuées en séparant les données relatives aux filles et aux garçons. Ensuite deux 
traitements multi-groupes ont été planifiés pour obtenir un modèle de base sans contrainte d'égalité et un modèle d'invariance totale des paramètres entre les deux groupes.

Le tableau 2 permet de constater que les indices d'ajustement du modèle sont acceptables pour les filles et pour les garçons, l'ajustement étant meilleur pour ces derniers. Ensuite, la structure à un seul facteur avec une covariation des erreurs pour les items 5 et 8 est testée simultanément sur les deux groupes selon deux modèles d'ajustement sur les paramètres. La différence d'ajustement entre le modèle sans contrainte et le modèle d'invariance n'est pas significative, $\chi^{2}$ diff $(21)=13,33$, le modèle structurel retenu est donc invariant sur le genre. Cette invariance légitime la comparaison des moyennes. Les filles présentent un score moyen de dépression supérieur à celui des garçons : 9,69 vs 7,19; $\mathrm{t}(267)=4,13, p<0,001$.

Tableau 2. Analyses multi-groupes et indices d'ajustement

\begin{tabular}{lccccc}
\hline Modèles & $\chi^{2}$ & $\mathrm{dl}$ & $\chi^{2} / \mathrm{dl}$ & RMSEA & CFI \\
\hline Analyses séparées & & & & & \\
$\quad$ Filles seulement & 67,088 & 34 & 1,973 & 0,079 & 0,867 \\
Garçons seulement & 40,891 & 34 & 1,203 & 0,038 & 0,962 \\
Analyses multi-groupes & & & & & \\
Base (pas de contraintes) & 107,978 & 68 & 1,588 & 0,063 & 0,908 \\
Invariance totale & 121,312 & 89 & 1,363 & 0,048 & 0,925 \\
\hline
\end{tabular}

\section{Discussion}

L'objectif principal de cette étude était de valider la version française de la CES-D10 auprès d'adolescents et de tester l'hypothèse d'une structure unidimensionnelle de cette version courte. En corollaire, nous souhaitions éprouver l'invariance de cette structure selon le genre.

La cohérence interne constitue une première indication de la qualité psychométrique de l'échelle. Pour la CES-D originale en 20 items, cette cohérence est généralement élevée indépendamment des caractéristiques des populations étudiées. Par exemple, pour les adolescents, Roberts et al. [23], ainsi que Chabrol et al. [7] pour la version française, 
obtiennent un indice supérieur à 0,85 . Nos résultats avec une version courte sont moins bons avec un alpha de 0,75 qui reste cependant acceptable. L'écart peut s'expliquer en partie par le fait que l'alpha a tendance à augmenter avec le nombre d'items. Pour des versions courtes de la CES-D, on trouve généralement des alphas entre 0,70 et 0,75 [3, 15, 17, 25].

De nombreuses recherches ont confirmé la structure en quatre facteurs de la CES-D en 20 items, y compris chez des adolescents français [7]. Dans l'élaboration de versions courtes, les auteurs ont cherché à recomposer cette structure, hormis la composante Problèmes interpersonnels. Les recherches aboutissent à deux types de conclusions : la persistance d'un modèle à trois facteurs (Affect dépressif, Troubles somatiques et Affect positif) avec un facteur de second ordre de symptomatologie dépressive $[8,9]$ ou un modèle bi-factoriel Affect négatif (regroupant les composantes fortement corrélées Affect dépressif et Troubles somatiques) et Affect positif [3, 4, 17]. Notre positionnement a été différent: nous avons considéré que l'intérêt d'une version courte n'est pas de fournir à l'utilisateur un score par sous-échelle afin d'établir un diagnostic différencié mais de proposer un seul score indicateur d'un positionnement sur l'échelle. Trois arguments plaident en faveur de cette perspective. Tout d'abord, en dépit de la confirmation d'une structure multi-dimensionnelle, de nombreux auteurs recommandent l'utilisation du score global sur l'ensemble des items [13, 15, 21]. Le deuxième argument tient à la faible fiabilité du facteur Affect positif: deux items sur une échelle de réponse en quatre points sont insuffisants pour garantir une bonne interprétation du score partiel. Les alphas obtenus dans notre recherche $(\alpha=0,38)$ comme dans celle de Bradley, Bagnell et Brannen [4] $(\alpha=0,40)$ confirment ce défaut de cohérence interne. Enfin, dans la ligne des écrits d'autres auteurs [15, 27, 28], le facteur Affect positif pourrait être en réalité un artefact méthodologique, les deux items qui le composent étant les seuls formulés avec une orientation positive. En définitive, sauf à constater l'insuffisance d'ajustement du modèle unidimensionnel autorisant la corrélation des erreurs associées aux deux items d'Affect positif, l'intérêt de la version courte est de fournir un indicateur unique permettant des comparaisons inter et intra-individuelles aisées. Nos résultats vont dans ce sens et, à notre connaissance, l'hypothèse de l'artefact n'avait pas été testée pour la population adolescente.

De même, nous ne connaissons pas d'études sur la version courte de la CES-D ayant vérifié l'invariance structurelle selon le genre pour la population adolescente. Or cette vérification est absolument nécessaire pour éviter un biais d'interprétation dans les comparaisons entre les filles et les garçons. Ce biais avait été signalé dans le cas de la version à 20 items. Lee et Chokkanathan [17], avec la CES-D10 et pour une population de personnes âgées, ont 
confirmé l'invariance selon le genre pour un modèle à deux facteurs. Van de Velde et al. [28], quant à eux, ont établi l'invariance selon le genre pour une version en huit items et un modèle unifactoriel avec covariation des erreurs associées aux items d'Affect positif. Nos résultats vont donc dans le même sens et indiquent que les items utilisés se réfèrent à une même dimension psychologique pour les adolescentes et les adolescents. La comparaison des moyennes entre ces deux groupes révèlent un écart significatif de 2,5 points en défaveur des filles. Chez des lycéens français, Chabrol et al. [7] avaient constaté un écart d'un peu plus de 4,2 points pour la version en 20 items.

En conclusion, la version française de la CES-D10 constitue un instrument possédant des qualités psychométriques suffisantes pour être utilisé auprès d'une population d'adolescents, dès 14 ans. Pour les praticiens, l'échelle peut ainsi être utilisée comme outil de dépistage de première intention $[18,24]$. Pour les chercheurs, l'échelle peut être, compte tenu de sa brièveté, plus facilement associée à d'autres instruments permettant ainsi des investigations à large spectre des comportements de santé des adolescents. L'invariance structurelle selon l'âge reste un objectif de recherche important pour permettre des études longitudinales. 


\section{Références}

[1] Andresen EM, Malmgren JA, Carter WB, et al. Screening for depression in well older adults: Evaluation of a short form of the CES-D (Center for Epidemiologic Studies Depression scale). Am J Prev Med 1994; 10(2): 77-84.

[2] Beals J, Manson SM, Keane EM, et al. Factorial structure of the Center for Epidemiologic Studies - Depression Scale among American Indian college students. Psychol Assess 1991; 3(4): 623-27.

[3] Boey KW. Cross-validation of a short form of the CES-D in Chinese elderly. Int J Geriatr Psychiatry 1999; 14(8): 608-17.

[4] Bradley KL, Bagnell AL, Brannen CL. Factorial validity of the Center for Epidemiological Studies Depression 10 in adolescents. Issues Ment health nurs 2010; 31(6): 408-12.

[5] Browne MW, Cudeck R. Alternative ways of assessing model fit. Sociol Method Res 1992; 21(2): 230-58.

[6] Callahan CM, Wolinsky FD. The effect of gender and race on the measurement properties of the CES-D in older adults. Med Care 1994; 32(4): 341-56.

[7] Chabrol H, Montovany A, Chouicha K, et al. Study of the CES-D on a sample of 1953 adolescent students. Encéphale 2002; 28: 429-32.

[8] Cheng ST, Chan ACM, Fung HH. Factorial structure of a short version of the Center for Epidemiologic Studies-Depression scale. Int J of Geriatr Psychiatry 2006; 21(4): 333-36.

[9] Cole JC, Rabin AS, Smith TL, et al. Development and validation of a Rasch-derived CES-D short form. Psychol Assess 2004; 16(4): 360-72.

[10] Diener E, Larsen RJ, Levine S, et al. Intensity and frequency: Dimensions underlying positive and negative affect. J Pers Social Psychol 1985; 48(5): 1253-65.

[11] Fuhrer R, Rouillon F. La version française de l'échelle CES-D (Center for Epidemiologic Studies-Depression Scale). Description et traduction de l'échelle d'auto-évaluation. Psychiatr Psychobiol 1989; 4(3): 163-66.

[12] Garrison CZ, Addy, CL, Jackson KL, et al. The CES-D as a screen for depression and other psychiatric disorders in adolescents. J Am Acad Child Adolesc Psychiatry 1991; 30(4): 636-41.

[13] Hertzog C, Van Alstine J, Usala PD, et al. Measurement properties of the Center for Epidemiological Studies Depression Scale (CES-D) in older populations. Psychol Assess 1990; 2(1): 64-72.

[14] Hu LT, Bentler PM. Cut-off criteria for fit indexes in covariance structure analysis: Conventional criteria versus new alternatives. Structural Equation Modeling 1999; 6(1): $1-55$.

[15] Kohout JF, Evans AD, Cornoni-Huntley J. Two shorter forms of the CES-D Depression 
Symptoms Index. J Aging Health 1993; 5(2): 179-93.

[16] Kline RB. Principles and practice of structural equation modeling, $2^{\text {nd }}$ Edition. The Guilford Press: London; 2005.

[17] Lee A, Chokkanathan S. Factor structure of the 10-item CES-D10 scale among community dwelling older adults in Singapore. Int J Geriatr Psychiatry 2008; 23(6): 59297.

[18] Lewinsohn PM, Hoberman HH, Rosenbaum M. A prospective study of risk factors for unipolar depression. J Abnorm Psychol 1988; 97(3): 251-64.

[19] Nolen-Hoeksema S, Girgus J. The emergence of gender differences in depression during adolescence. Psychol Bull 1994; 115(3): 424-43.

[20] Pelkonen M, Marttunen M, Kaprio J, et al. Adolescent risk factors for episodic and persistent depression in adulthood. A 16-year prospective follow-up study of adolescents. J Affect Disord 2008; 106(1): 123-31.

[21] Radloff LS. The CES-D scale: A self report depression scale for research in the general population. Appl Psychol Meas 1977; 1(3): 385-401.

[22] Radloff LS. The use of the Center for Epidemiologic Studies-Depression scale in adolescents and young adults. J Youth Adol 1991; 20(2): 149-66.

[23] Roberts RE, Andrews JA, Lewinsohn PM, et al. Assessment of depression in adolescents using the Center for Epidemiologic Studies Depression Scale. Psychol Assess 1990; 2(2): 122-28.

[24] Santor DA, Coyne JC. Shortening the CES-D to improve its ability to detect cases of depression. Psychol Assess 1997; 9(3): 233-43.

[25] Séjourné N, van Leeuwen N, Sobolewski G, et al. Les contributions relatives de la symptomatologie dépressive, du lien d'attachement aux parents et de l'impact négatif des événements de vie, au syndrome limite de l'adolescent. Neuropsychiatr Enf Adolesc 2009; 57: 368-71.

[26] Stommel M, Given, BA, Given CW, et al. Gender bias in the measurement properties of the Center for Epidemiologic Studies Depression Scale (CES-D). Psychiatry Res 1993; 49(3): 239-50.

[27] Turvey LC, Wallace BR, Herzog, R. A revised CES-D measure of depressive symptoms and a DSM-based measure of major depressive episodes in the elderly. Int Psychogeriatr 1999; 11(2): 139-48.

[28] Van de Velde S, Bracke P, Levecque K, et al. Gender differences in depression in 25 European countries after eliminating measurement bias in the CES-D8. Soc Sci Res 2010; 39(3): 396-404.

[29] Wild LG, Fisher AJ, Lombard C. Suicidal ideation and attempts in adolescents: associations with depression and six domains of self-esteem. J Adolesc 2004; 27(sup. 6): 611-24. 


\section{Annexe}

Items de la CES-D10

Consigne : Les impressions suivantes sont ressenties par la plupart des gens. Peux-tu indiquer si tu les as ressenties souvent pendant la semaine écoulée?

Jamais, très rarement (moins d'un jour) ;

Occasionnellement (1 à 2 jours)

Assez souvent (3 à 4 jours) ;

Fréquemment, tout le temps (5 à 7 jours).

1/ J'ai été contrarié(e) par des choses qui d'habitude ne me dérangent pas

2/ J'ai eu du mal à me concentrer sur ce que je faisais

3/ Je me suis senti(e) déprimé(e)

4/ J'ai eu l'impression que toute action me demandait un effort

5/ J'ai été confiant(e) en l'avenir

6/ Je me suis senti(e) craintif(ve)

7/ Mon sommeil n'a pas été bon

8/ J'ai été heureux(se)

9/ Je me suis senti(e) seul(e)

10/ J'ai manqué d'entrain 